\title{
CRIB (clinical risk index for babies) in relation to nosocomial bacteraemia in very low birthweight or preterm infants
}

\author{
PW Fowlie, C R Gould, G J Parry, G Phillips, W O Tarnow-Mordi
}

\begin{abstract}
Positive blood cultures in very low birthweight or preterm infants usually reflect bacteraemia, septicaemia, or failure of asepsis during sampling and lead to increased costs and length of stay. Rates of nosocomial, or hospital acquired, bacteraemia may therefore be important indicators of neonatal unit performance, if comparisons are adjusted for differences in initial risk.

In a preliminary study the risk of nosocomial bacteraemia was related to initial clinical risk and illness severity measured by the clinical risk index for babies (CRIB). Nosocomial bacteraemia was defined as clinically suspected infection with culture of bacteria in blood more than 48 hours after birth.

One or more episodes of noscomoial bacteraemia were identified retrospectively in 36 of $143(25 \%)$ infants in a regional neonatal unit between 1992 and 1994. Biologically plausible models were developed using regression analysis techniques. After correcting for period at risk, nosocimial bacteraemia was independently associated with gestation at birth and CRIB. Death was independently associated with CRIB, but not with noscomial bacteraemia.

CRIB may contribute, with other explanatory variables, to more comprehensive predictive models of death and nosocomial infection. These may facilitate future risk adjusted comparative studies between groups of neonatal units. (Arch Dis Child 1996;75:F49-F52)
\end{abstract}

Keywords: nosocomial septicaemia, league tables, institutional performance, CRIB.

Mortality is not the only indicator of performance in neonatal intensive care. Nosocomial (hospital acquired) infections of blood, respiratory tract, urine, brain, bone and wounds occur in up to $60 \%$ of infants in intensive care and are dangerous, costly, and partly preventable. ${ }^{1}$ Variation in the incidence of nosocomial infection among neonatal intensive care units ${ }^{2}$ may reflect relative overcrowding, understaffing, ${ }^{3-5}$ early feeding or poor aseptic practice, all of which are indicators of of care or case mix.

Crude comparisons of nosocomial infections are subject to confounding, however, because of substantial differences among institutions in birthweight, gestational age, and criteria and techniques used for the investigation and diagnosis of infection, the use of indwelling catheters and the duration of exposure to risk as reflected by length of stay. ${ }^{2}{ }^{6}$ Recently, initial illness severity up to 24 hours after admission, as measured by SNAP (score for acute neonatal physiology), was an important confounding variable in assessing the risk of nosocomial bacteraemia. $^{7}$

Diagnostic bias is a major problem in comparisons between institutions. Positive blood cultures obtained more than 48 hours after admission are less prone to inter- and intra-observer bias than are the diagnoses of pneumonia or wound infection, and they provide a common, accurate, and important measure of clinical performance. They usually indicate bacteraemia or septicaemia acquired in hospital, or poor sampling technique, and lead to prolonged antibiotic treatment and repeated investigations, with increased costs and length of stay. ${ }^{7}$

The incidence of positive blood cultures may, therefore, provide a reliable composite indicator of nosocomial bacteraemia or poor aseptic sampling technique, but this statistic is not routinely available. Comparative data are also likely to be misleading if they are not adjusted for differences in case mix, or initial risk. We assessed whether CRIB (clinical risk index for babies) ${ }^{8} 9$ can adjust for differences in clinical risk and initial illness severity up to 12 hours after birth when reporting the risk of bacteraemia in very low birthweight or preterm infants. Our main hypothesis was that the risk of bacteraemia was associated with initial clinical risk and illness severity, as measured by CRIB, after correcting for length of stay. The secondary hypotheses were that after adjustment for initial illness severity, the risk of death and total length of stay (in survivors) were independently associated with nosocomial bacteraemia.

\section{Methods}

This was an observational study in a cohort of infants admitted to Ninewells Hospital Special Care Baby Unit between 1 January 1992 and 31 December 1994. The unit is a regional centre serving Tayside, Scotland, with roughly 350 admissions a year. Surfactant was in routine use and more than $60 \%$ of women delivering before 31 weeks of gestation received antenatal steroids during the study period. All infants admitted with a birthweight under $1501 \mathrm{~g}$ or a 
Table 1 Characteristics of the study infants $(n=143)$

\begin{tabular}{lcl}
\hline & Median & Range \\
\hline Weight (g) & 1260 & $600-1870$ \\
Gestation (completed weeks) & 30 & $25-36$ \\
CRIB score & 1 & $0-13$ \\
Length of stay (days) & 35 & $2-130$ \\
\hline
\end{tabular}

Table 2 Organisms isolated during study period

\begin{tabular}{ll}
\hline Organism & $\begin{array}{l}\text { Total number of episodesof } \\
\text { nosocomial bacteraemia }\end{array}$ \\
\hline Staphylococcus epidermidis & 32 \\
Staphylococcus aureus & 1 \\
Enterococcus spp & 4 \\
Klebsiella spp & 2 \\
Eschericha coli & 7 \\
Group B streptococcus & 1 \\
\hline
\end{tabular}

gestational age under 31 weeks, and who remained in the unit beyond 48 hours of age, were included. Data were collected retrospectively from clinical records by two research assistants and included initial clinical risk and illness severity as measured by CRIB, length of stay, mortality, the number of episodes of bacteraemia, and the bacteria isolated. An episode of bacteraemia was defined as clinically suspected sepsis presenting later than $\mathbf{4 8}$ hours after birth associated with growth of bacteria on blood culture. Episodes were considered to be distinct and counted separately if positive blood cultures were at least five days apart. Blood cultures were never obtained without clinical suspicion of infection. Length of stay was defined as the time in days between admission to the unit and either death or discharge from the unit to another ward, another hospital, or to home. The data collectors were unaware of the hypotheses being tested and those responsible for interpreting the blood cultures were unaware of the clinical condition of each infant. Approval for the study was sought from the Tayside Committee on Medical Research Ethics before data collection and deemed not necessary.

STATISTICAL METHODS

Survival analysis techniques were used to examine the relation between time to first occurrence of bacteraemia and possible explanatory variables, including birthweight, gestational age at birth, and initial risk and illness severity (as measured by CRIB). Using Cox regression with forward stepwise methods, a model relating time to first occurrence of bacteraemia to initial disease severity was obtained. Similar methods were used to examine the correlation between time to hospital mortality and the occurrence of bacteraemia, after adjusting for differences in initial risk and illness severity using CRIB. Finally, the relation between length of stay after correcting for differences in gestation at birth, the occurrence of bacteraemia, and initial illness severity as measured by CRIB, was examined using the same methods. Those variables whose contribution to any given model did not reach the significance of $P<0.05$ for their regression coefficient were dropped from the model. SPSS version 6.1.2 software was used throughout (SPSS Inc., Chicago, Illinois, USA).

\section{Results}

Ninety nine per cent of eligible infants were included in the study ( 77 boys and 66 girls). Two infants were excluded from the analyses because of incomplete data. The characteristics of the cohort are shown in table 1 and figure 1 (A-C). One hundred and seven infants had no recorded episode of bacteraemia up until their death or discharge from the unit; 26 infants had a single episode of bacteraemia; seven had two episodes of infection; and three infants had three episodes. The pathogens isolated are shown in table 2 . Seventeen infants died during their admission to the unit.

\section{NOSOCOMIAL BACTERAEMIA}

CRIB and gestational age at birth were related independently to time to occurrence of bacteraemia. Examination of the residuals suggested
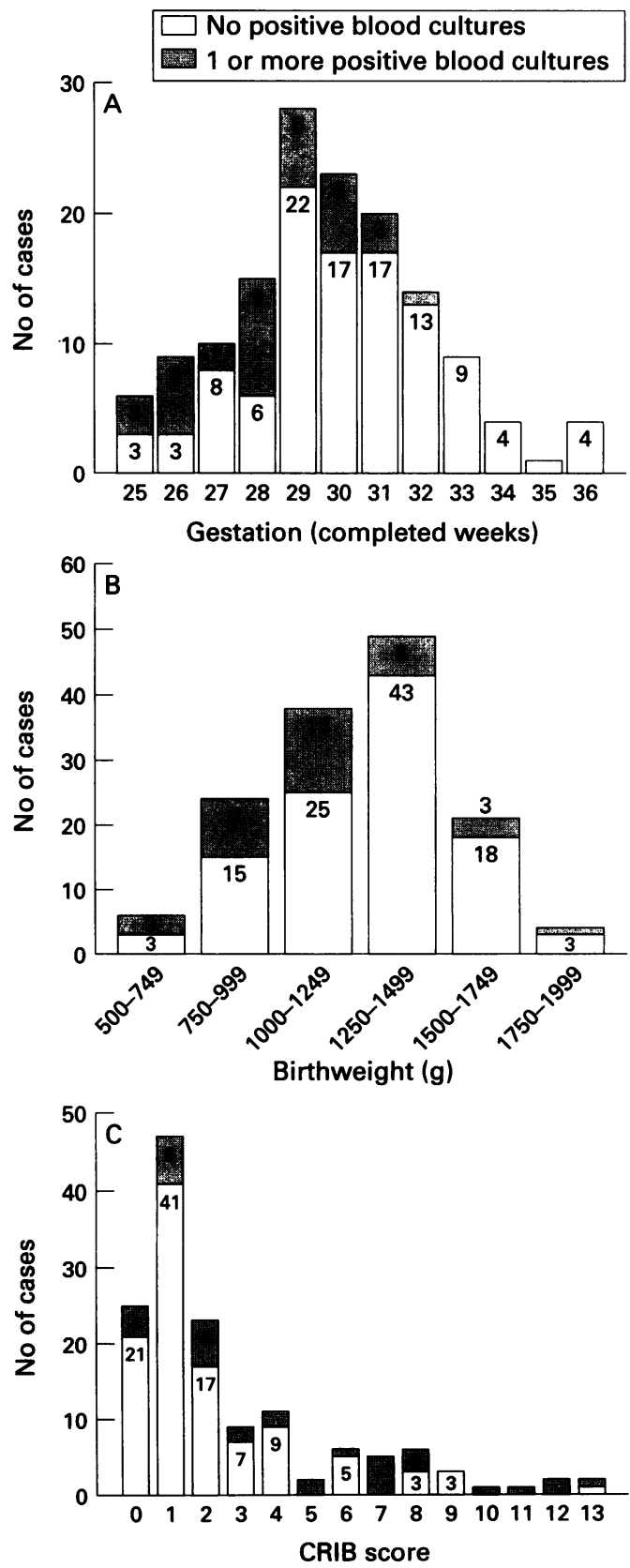

Figures 1A-C Proportion of study population ( $n=143$ ) with one or more episodes of nosocomial bacteraemia: $(A)$ frequency distribution of gestational age at birth; $(B)$ frequency distribution of gestational age at birth; (B) distribution of CRIB score. 
that this model fitted the data adequately and was valid. The model suggested that, for any given baby, at any given time, the risk of one or more episodes of nosocomial bacteraemia increased by a factor of 1.12 (95\% confidence interval $1.01,1.24$ ) for each unit increase in CRIB and changed by a factor of $0.80(95 \% \mathrm{CI}$ $0.67,0.96)$ for each increase in one week of gestation at birth. Figure 2A illustrates the independent relation between CRIB and time to occurrence of bacteraemia. Figure 2B illustrates the independent relation between gestational age at birth and time to occurrence of bacteraemia.

DEATH

Of the four variables (CRIB, gestational age at birth, birthweight and the occurrence of one or more episodes of bacteraemia) entered into the model for risk of death, only CRIB was independently related: for every unit increase in CRIB score, the risk of mortality increased by a factor of 1.32 ( $95 \%$ confidence interval $1.16,1.49$ ).

\section{LENGTH OF STAY}

In those infants who survived to hospital discharge, total length of stay was independently related to the occurrence of one or more episodes of bacteraemia, CRIB, and gestational
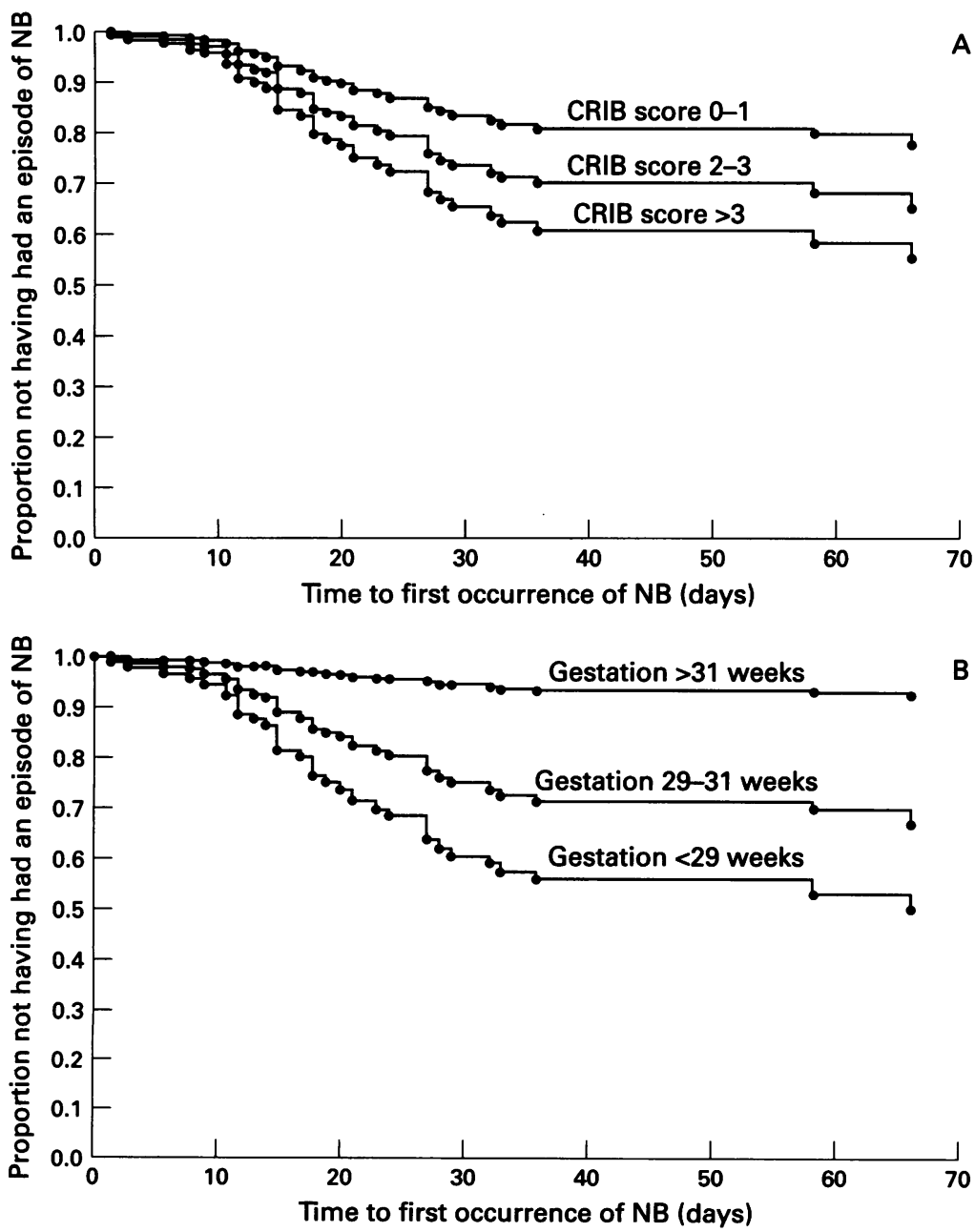

Figure 2 Chances of not having had an episode of nosocomial bacteraemia (NB) at any given time following admission: (A) risk of nosocomial bacteraemia, time after admission, and CRIB; (B) risk of nosocomial bacteraemia, time after admission, and gestational age at birth. age at birth. Thus for each survivor, at any given time, the chance of an extra day's stay was increased independently by a factor of 1.19 (95\% confidence interval $1.08,1.32$ ) for each unit increase in CRIB, by a factor of 2.27 (95\% confidence interval $1.40,3.67$ ) if the infant had one or more episodes of bacteraemia, and by a factor of 0.79 ( $95 \%$ confidence interval $0.73,0.86$ ) for each additional week of gestation at birth.

\section{Discussion}

The main hypothesis, that initial clinical risk and illness severity measured by CRIB is independently associated with the risk of nosocomial bacteraemia, is accepted. Although early mortality may result in misleadingly low rates of bacteraemia in some centres, by curtailing the duration of exposure to risk, this potential bias was overcome using survival analysis with Cox regression analysis. An alternative strategy would have been to develop a predictive model for the composite outcome of death or bacteraemia before discharge. When comparing the incidence of nosocomial bacteraemia, measures of risk such as CRIB and gestational age at birth may be important explanatory variables, among others. Risk adjusted comparisons are, therefore, likely to be more valid. When adjusting for differences in illness severity between centres, CRIB may offer advantages over more complex scoring systems. CRIB is a validated measure of initial clinical risk and illness severity up to 12 hours after birth, ${ }^{8-10}$ but contains only six variables and is simple to calculate.

There was no evidence to support the hypothesis that the risk of death was independently related to nosocimial bacteraemia. This is plausible given that most episodes are not regarded as life-threatening. When birthweight and gestational age were added to this model as independent variables, neither entered the final equation. Death was therefore still predicted more accurately by CRIB than by birthweight or gestation alone, confirming the findings of a recent study. ${ }^{10}$ This supports the continued use of CRIB, which was originally validated before the introduction of surfactant treatment, ${ }^{8}$ to adjust for case mix now that surfactant is in routine use. As might be expected clinically, initial illness severity, the occurrence of one or more episodes of bacteraemia, and gestational age at birth significantly and independently contributed to length of stay among surviving infants. Larger numbers are, however, needed to develop and validate more comprehensive models for length of stay, to adjust for case mix in comparative multicentre studies.

The number of confounding variables examined in the analyses was limited. Others probably exist, but their independent impact on outcome is unknown. In particular, invasive treatments such as central venous catheters may increase the risk of bacteraemia. Simply adjusting interhospital comparisons as infection rates per 100 catheter days at risk ${ }^{1}$ masks variability in clinical preferences for invasive support and may therefore overcontrol for differences in policy. It is also plausible that the 
number of cotside visits might, in itself, also be associated with the risk of nosocomial bacteraemia and there is now evidence to suggest that sicker infants are likely to receive more cotside visits (W Tarnow-Mordi, K Hamilton, C Gould, G Parry, P Fowlie for the Scottish Neonatal Consultants Collaborative Study Group and the International Neonatal Network. Abstract presented to the British Association of Perinatal Medicine, September 1995). Bacteraemia or sepsis from endogenous bacterial translocation across the gut may be increased by insults such as hypoxia or hypothermia, ${ }^{11}$ which correlate with severity of illness, and are reduced by milk ${ }^{12}$ and trophic feeding (McClure RJ, Newell SJ. Trophic feeding, gastrointestinal motility and clinical outcome in the preterm infant receiving intensive care. Abstract presented to the British Association, April 1996). Lastly, venous access may become progressively more difficult in sicker infants, increasing the risks of either introducing infection through repeated skin punctures, or of positive blood cultures representing growth of skin contaminants. Accurate estimation of initial risk of bacteraemia would allow differences between observed and expected rates to be attributed to variations in clinical policy.

Although the definition of nosocomial bacteraemia used in this study is widely accepted, ${ }^{13}$ a few hospital acquired infections occur before 48 hours ${ }^{14}$ and a few positive cultures arising after 48 hours reflect vertically transmitted septicaemia. As such cases are rare, they will have little impact on the validity of multicentre comparisons but it would be reasonable to record the spectrum of organisms isolated in each neonatal unit.

Because of the relatively small numbers treated in individual neonatal units, "league tables" ranking each unit by their rate of bacteraemia or mortality are likely to be misleading. ${ }^{15}$ Even after adjusting for differences in initial risk, using highly accurate predictive models, comparisons between individual institutions will generate wide confidence intervals around point estimates of observed or expected outcome. Consequently, substantial fluctuations in individual rankings should be expected purely by chance alone. ${ }^{15}$ Comparisons of risk adjusted outcome between groups of institutions may also be inconclusive if based on small numbers. ${ }^{10}$ The impact of organisational characteristics on risk adjusted outcome can only be studied successfully using adequate sample sizes. ${ }^{16}$
Nosocomial bacteraemia is a common, costly, and clinically important measure of outcome and may be a valuable indicator of performance in neonatal intensive care. A large multicentre study is needed to develop and validate a predictive model for bacteraemia that will adjust for variations in case mix when comparisons are made between groups of units. Initial severity of illness, as measured by CRIB, may contribute to such a model.

$P$ Fowlie is an MRC Training Fellow in Health Services Research. C Gould and the research assistants were supported by the Wellcome Trust and the Scottish Office Clinical Resource and Audit Group (CRAG).

Dr D Richardson and Professor B Schmidt provided valuable comments.

1 Josephson A, Karanfil L, Alonso H, Watson A, Blight J. Riskspecific nosocomial infection rates. $A m \neq$ Med 1991; 91(suppl 3B): 131S-7S.

2 Gaynes RP, Martone WJ, Culver DH, Emori TG, Horan TC, Banerjee SN. Comparison of rates of nosocomial infection in neonatal intensive care units in the United States. Am $\mathcal{F}$ Med 1991;91:192-6S.

3 Goldmann D, Durbin W, Freeman J. Nosocomial infections in a neonatal intensive care unit. $\mathcal{F}$ Infect Dis 1981;144:44959.

4 Haley RW, Bregman DA. The role of understaffing and overcrowding in recurrent outbreaks of staphylococcal infection in a neonatal special care unit. $\mathcal{f}$ Infect Dis 1982; 145:875-85.

5 Isaacs D, Catterson J, Hope PL, Moxon ER, Wilkinson AR. Factors influencing colonisation with gentamicin resistant Factors influencing colonisation with gentamicin resistant Gram negative organism

6 Freeman J, Platt R, Epstain MF, Smith NE, Sidebottom DG, Goldmann DA. Birthweight and length of stay as determinants of nosocomial coagulase-negative staphylococcal bacteremia in neonatal intensive care unit populations: potential for confounding. Am $₹$ Epidemiol 1990 132:1130-40.

7 Gray JE, Richardson DK, McCormick MC, Goldmann DA. Coagulase-negative staphylococcal bacteremia among very low birthweight infants: relation to admission illness severity, resource use and outcome. Pediatrics 1995;95:225-30.

8 The International Neonatal Network. The CRIB (clinical risk index for babies) score: a tool for assessing initial neorisk index for babies) score: a tool for assessing initial neonatal risk and comparing performance

9 Scottish Neonatal Consultants' Collaborative Study Group and the International Neonatal Network. CRIB (clinical risk index for babies), mortality, and impairment after neonatal intensive care. Lancet 1995;345:1020-2

10 de Courcy-Wheeler RHB, Wolfe CDA, Fitzgerald A, Spencer M, Goodman JDS, Gamsu HR. Use of the CRIB (clinical risk index for babies) score in prediction of neonatal mortality and morbidity. Arch Dis Child 1995; 73:F32-6.

11 Van Camp JM, Tomaselli V, Cotan AG. Bacterial translocation in the neontate. Curr Opin Pediatr 1994;6:327-33.

12 Albanese CT, Watkins SC, Simmons RL, Rowe MI. Breast milk protects the neonate from bacterial translocation. Pediatr Surg 1994;29:1059-64.

13 Isaacs D. Hospital acquired infections. In: De Louvois J, Harvey $\mathrm{D}$, eds. Infection in the newborn. London: John Wiley, 1990:35-47.

14 Stoll BJ, Fanaroff A. Early onset coagulase-negative staphylococcal sepsis in preterm neonate. National Institute of Child Health and Human Development (NICHD) Neonatal Research Network. Lancet 1995; 345:1236-7.

15 Goldstein H, Spiegelhalter DJ. League tables and their limitations: statistical issues in comparisons of institutional performance. Series A. $\mathcal{F} R$ Stat Soc (in press).

16 Pollack MM, Cuerdon TT, Patel KM, Ruttiman UE, Getson PR, Levetown M. Impact of quality of care factors on pediatric intensive care unit mortality. $¥ A M A 1994$; 272:941-6. 